\title{
Failed Completion Pneumonectomy for Early Recurrent Pleomorphic Carcinoma: A Case Report ${ }^{*}$
}

\author{
Mayuko Uehara", Atsushi Watanabe, Junji Nakazawa, Ryo Harada, Masahiro Miyajima, \\ Shinji Nakashima, Takuro Obama, Tetsuya Higami
}

Department of Thoracic and Cardiovascular Surgery, Sapporo Medical University, Sapporo, Japan.

Email: "mayuko@sapmed.ac.jp

Received October $9^{\text {th }}, 2012$; revised November $12^{\text {th }}, 2012$; accepted November $24^{\text {th }}, 2012$

\begin{abstract}
We report a patient with a large pulmonary pleomorphic carcinoma (PPC) in the left upper lobe greater than $10 \mathrm{~cm}$ in diameter. She underwent left upper lobectomy with mediastinal lymph node dissection and concomitant resection of the left phrenic nerve, vagus nerve and pericardium. She received adjuvant chemotherapy, but had tumor recurrence. We then performed left completion pneumonectomy, but could not remove the tumor completely because of rapid tumor invasion to the left atrium. Urgent surgery should be considered for recurrent resectable PPC.
\end{abstract}

Keywords: Pulmonary Pleomorphic Carcinoma; Completion Pneumonectomy; Reoperation; Tumor Recurrence

\section{Introduction}

In the World Health Organization histologic classification of lung tumors, pulmonary pleomorphic carcinoma (PPC) is classified as a new histologic subtype [1]. Because of its rare occurrence, it has been little reported, and only a few case reports or clinical reviews have been published [2-4]. We report a patient in whom rapid tumor progression developed despite two operations.

\section{Case Report}

A 58-year-old woman with a history of cigarette smoking, and no marked medical or family history, presented with persistent general fatigue. A computed tomography (CT) showed a large upper lobe tumor, greater than $10 \mathrm{~cm}$ in diameter, occupying the anterior mediastinum (Figure 1). She was given a diagnosis of lung cancer invading into the anterior mediastinum.

Median subtotal sternotomy and fourth intercostal anterolateral thoracotomy were performed. The tumor originated from the left upper lobe and involved the left phrenic nerve, the vagus nerve and the pericardium. The pericardium was opened longitudinally and the left main pulmonary artery taped in order to clamp it any time if necessary. Left upper lobectomy was performed with mediastinal lymph node dissection and concomitant re-

*Funding: This research received no specific grant from any funding agency in the public, commercial, or not-for-profit sectors. Conflicts of interest statement: None declared.

${ }^{\#}$ Corresponding author. section of the left phrenic nerve, the vagus nerve proximal to the origin of the recurrent laryngeal nerve, and the pericardium. She was discharged from hospital 10 days after surgery.

Histopathological findings revealed that more than $90 \%$ of the tumor comprised proliferating spindle cells, and malignant squamous cells were scattered throughout the tumor (Figure 2). Additionally, the tumor was revealed as PT4NOM0 stage IIIA, with no vascular invasion. She was given a diagnosis of PPC in the left upper lobe of the lung.

She received standard adjuvant chemotherapy $[5,6]$ for non-small cell lung cancer (NSCLC) with cisplatin (100 $\mathrm{mg} / \mathrm{m}^{2}$ on day 1 ) and vinorelbine $\left(30 \mathrm{mg} / \mathrm{m}^{2}\right.$ on days 1 and 8). Mild renal dysfunction occurred after the first course of adjuvant chemotherapy, so the regimen was changed to second-line therapy consisting of carboplatin (400 $\mathrm{mg} / \mathrm{m}^{2}$ on day 1 ) and paclitaxel $\left(210 \mathrm{mg} / \mathrm{m}^{2}\right.$ on day 1).

Four months after the operation, a CT showed local tumor recurrence on the left bronchial stump (Figure 3(A)). A CT, 30 days after the recurrence showed that the tumor had enlarged very rapidly.

We performed left completion pneumonectomy by the same approach as before. However, the tumor could not be removed completely because of invasion to the left atrium. She was discharged after an uneventful recovery 16 days after the second operation, without any adjuvant chemotherapy or radiotherapy because she rejected further treatment and desired only best supportive care. A 


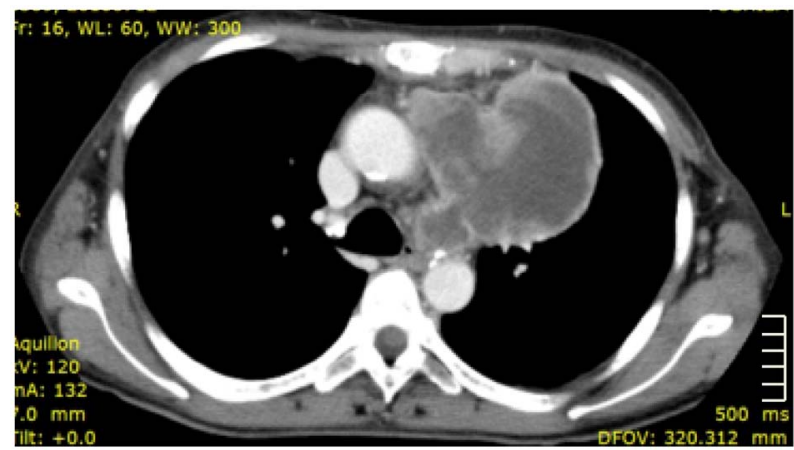

Figure 1. Computed tomography (CT) scan showing a large tumor (diameter $>10 \mathrm{~cm}$ ) occupying the anterior mediastinum.

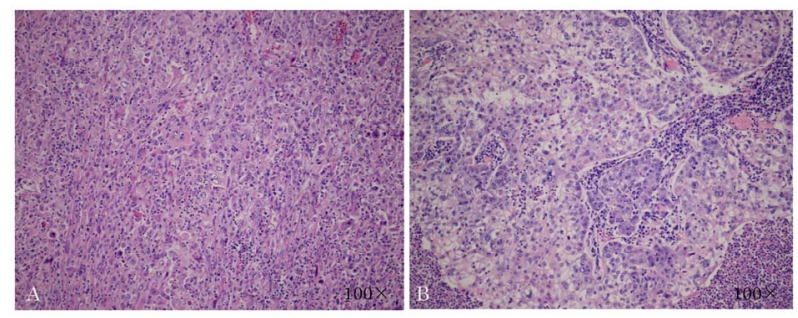

Figure 2. Histopathological findings. (A) Spindle cells; (B) Squamous cells.

CT, 1 month later showed the remnant of the tumor growing again (Figure 3(B)). She died on 4 months after 2nd surgery because of intrapericardial rapid tumor growing.

\section{Discussion}

Pleomorphic carcinoma of the lung is a rare tumor [4]. A few reports suggested that PPC tends to occur in males, smokers and the upper lobe [2-4]. When a patient visits a doctor, the tumor is often already very large, with metastasis. As a result, complete resection of PPC is sometimes difficult to perform.

Our patient's tumor size was over $10 \mathrm{~cm}$ in diameter, but we performed histopathologically complete tumor resection in the first operation. However, the tumor recurred locally postoperatively. According to a study by Yuki [3], there were statistically significant differences between pN0 and pN1/N2 groups in disease-free interval in PPC patients with surgical excision. Even in patients with pN0 disease, one-third had a recurrence within 14 months and died within 18 months. These data showed that PPC could be fatal even at an early stage, unlike other NSCLCs. In our case, in spite of complete tumor resection and adjuvant chemotherapy, the tumor recurred also.

After recognizing tumor recurrence in our patient, there was a 1-month delay before the second operation. The tumor progressed very rapidly and invaded the left atrium during the period (Figure 4). In patients with

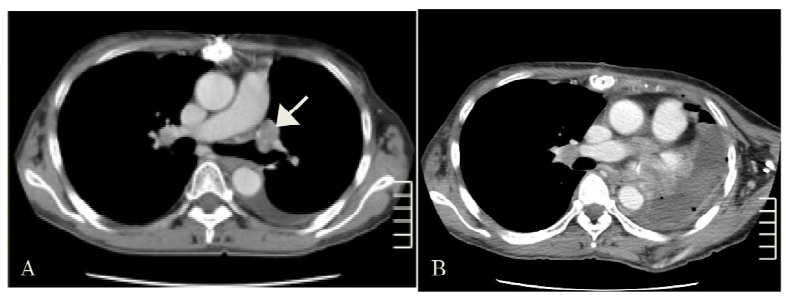

Figure 3. (A) 4 months after the first operation. CT showing tumor recurrence (arrow); (B) 1 month after the second operation. CT shows the remnant of the tumor growing.

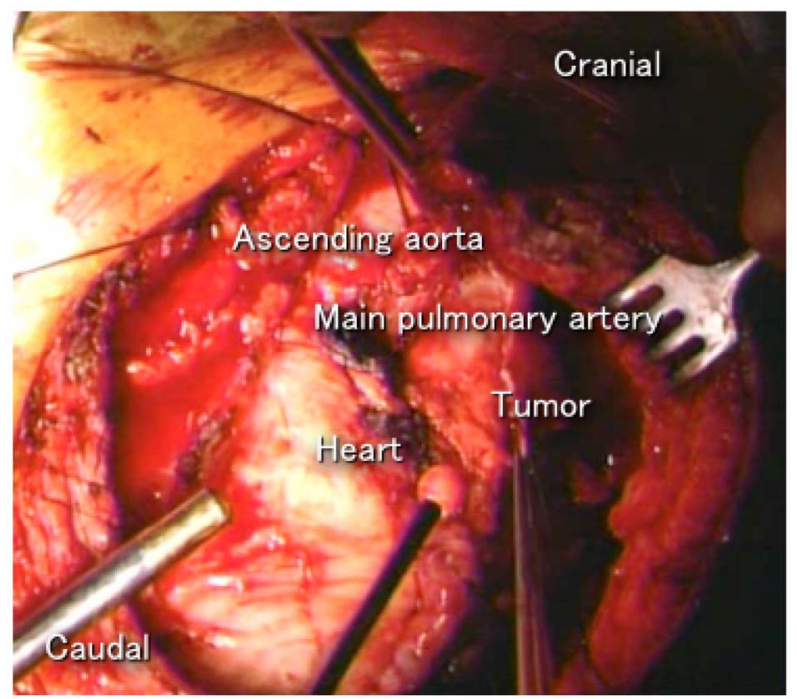

Figure 4. The tumor invaded the left atrium.

PPC, a 1-month interval from detection of local tumor recurrence to redo surgery may be too long for potential tumor resectability. A more timely second operation might have enabled us to perform complete tumor resection.

We consider that complete resection is necessary for PPC patients to have a more favorable prognosis. Therefore, we suggest that surgical intervention should be performed as soon as possible after recognition of the tumor if it is resectable, because the outcome is poor when tumor progression occurs and the tumor becomes unresectable.

Unfortunately, we have little information on the treatment of PPC, much less the one underwent surgery twice, because only a few reports are available. So further clinical data collection is warranted.

\section{REFERENCES}

[1] E. Brambilla, W. D. Travis, T. V. Colby, B. Corrin and Y. Shimosato, "The New World Health Organization Classification of Lung Tumors,” European Respiratory Journal, Vol. 18, No. 6, 2001, pp. 1059-1068. doi:10.1183/09031936.01.00275301

[2] M. Uehara, A. Watanabe, T. Nakajima, S. Nakashima, T. 
Higami and N. Inoue, "Pulmonary Pleomorphic Carcinoma; an Investigation of Thirteen Cases,” Kyobu Geka, Vol. 62, No. 2, 2009, pp. 89-93.

[3] T. Yuki, T. Sakuma, C. Ohbayashi, M. Yohimura, N. Tsubota, Y. Okita, et al., "Pleomorphic Carcinoma of the Lung: A Surgical Outcome,” The Journal of Thoracic and Cardiovascular Surgery, Vol. 134, No. 2, 2007, pp. 399404. doi:10.1016/j.jtcvs.2007.04.018

[4] N. F. Fishback, W. D. Travis, C. A. Moran, D. G. Guinee Jr., W. F. McCarthy and M. N. Koss, "Pleomorphic (Spindle/Giant Cell) Carcinoma of the Lung. A Clinicopathologic Correlation of 78 Cases," Cancer, Vol. 73, No. 12, 1994, pp. 2936-2945.

doi:10.1002/1097-0142(19940615)73:12<2936::AID-CN
CR2820731210>3.0.CO;2-U

[5] J. Y. Hong, M. K. Choi, J. E. Uhm, M. J. Park, J. Lee, Y. H. Park, et al., "The Role of Palliative Chemotherapy for Advanced Pulmonary Pleomorphic Carcinoma,” Medical Oncology, Vol. 26, No. 3, 2009, pp. 287-291. doi:10.1007/s12032-008-9117-4

[6] K. W. Lee, Y. J. Kim, J. H. Kim, S. M. Bang, J. H. Chung and J. S. Lee, "Two Consecutive Cases of Platinum-Refractory Pulmonary Pleomorphic Carcinoma that Showed Dramatic Responses to MAID (Mensa, Doxorubicin, Ifosfamide and Dacarbazine) Chemotherapy,” Japanese Journal of Clinical Oncology, Vol. 41, No. 3, 2011, pp. 430433. doi:10.1093/jjco/hyq180 